\title{
Utilization of rotation-invariant uniform LBP histogram distribution and statistics of connected regions in automatic image annotation based on multi-label learning
}

\author{
Sen Xia ${ }^{1,2,3,6}$,Peng Chen ${ }^{4}$, Jun Zhang ${ }^{5}$, Xiaoping Li ${ }^{6}$, Bing Wang ${ }^{1,2,3, *}$ \\ ${ }^{1}$ S choolof ElectronicsandInformationEngineering, Tong jiUniversity, Shanghai, China \\ ${ }^{2}$ TheAdvancedRe searchInstituteof IntelligentS ensingNetwork, Tong jiUniversity, Shanghai, China \\ ${ }^{3}$ The KeyLaboratoryof EmbeddedS ystemandS erviceComputing, Tong jiUniver sity, Shanghai, China \\ ${ }^{4}$ Instituteof HealthS ciences, AnhuiUniversity, Hefei, Anhui, China \\ ${ }^{5}$ Collegeof ElectricalEngineeringandAutomation, AnhuiUniversity, He fei, Anhui, China \\ ${ }^{6}$ Facultyof ComputerEngineering, HuaiyinInstituteofT echnology, Huaian, China
}

\begin{abstract}
A method for automatic image annotation based on multi-feature fusion and multi-label learning algorithm was proposed in this paper. In the process of feature fusion, rotation-invariant uniform local binary pattern histogram distribution and counting of connected regions in image were extracted and utilized fully. Besides traditional n-order color moments and texture information, rotation-invariant uniform LBP histogram distribution, connected regions number, weighted histogram's integral were appended to image features which aided to improve the average precision. Based on multi-label learning k-nearest neighbor algorithm and Corel5k image data set, comparisons among different dimensional features combinations were made to show that the proposed method outperformed that of traditional one with only basic color moments and texture distribution. The average precision was showed to be improved from 0.2898 to 0.3954 in automatic image annotation in our experimental results.
\end{abstract}

Keywords: LBP;connected regions;histogram distribution;multi-label learning;automatic image annotation

\section{Introduction}

Automatic image annotation used for both classification and identification is emerging as one of the powerful means for automatically recognizing semantic labels of image [1,2]. Whether biometric identification or natural scene semantics from images, higher confidence is the final goal [3]. It is important to discover the relation between unique features of image and its correponding semantic labels $[4,5,6]$. In traditional research, image retrieval was mainly developed into three different branches. The first one is text retrieval with the help of manual annotation, the second one is CBIR (content-based image retrieval) based on low level features of image, such as colour and texture. The third and last one is automatic image annotation which can implement the function of image retrieval by transforming the content retrieval into text retrieval $[7,8,9]$. In CBIR, extraction of unique features contained in image is one of the essential steps $[10,11,12]$.

Because of the limitations inherently existed in metadatabased systems, i.e., the dependence on annotation quality and completeness, fusion of scale invariant features was often used to improve the recognition rate [13]. Usually, image data are stored in 2D matrices, where each point represents the location

\footnotetext{
${ }^{*}$ Corresponding author.

Email address: wangbing@ustc. edu (Bing Wang 1,2,3,
}

of the pixel and the color value. Image should be expressed briefly as a refined set of image features, which make it practical to retrieve desired image from a big collection [14]. Statistical features of image from information of color, texture, shape have been used as important parameters in image retrieving [15]. However, it showed that single image feature was far from retrieving the satisfied results [16, 17]. Multi-feature fusion method therefore based on image content itself gets the place of single feature gradually in image retrieving $[18,19]$. Efficient features extraction methods are essential in the process of obtaining multi-feature information contained in image itself. Li, Zheng and Huang made use of locally linear discriminant embedding method to implement efficient feature extraction for face recognition [20]. Zhao, Huang and Sun proposed a face recognition method based on multi-feature using a neural networks committee machine [21]. Among those features, both global image information and local image information for image segmentation [22] were fully utilized. Local ChanVese model method based on the techniques of curve evolution, local statistical function and level set is used for image segmentation by Wang, Huang and Xu [23]. Caicedo and Jaafar BenAbdallah etc. presented a method based on non-negative matrix factorization to generate multimodal image representations that integrate visual features and text information [1]. Krishnamoorthi etc. proposed a image retrieval method with weighted multifeature 
set based on multiresolution enhanced orthogonal polynomials model and genetic algorithm [18].

In order to reduce the semantics gap brought by the incompliance between the machine understanding of structural image difference by machine learning mechanism [24] and that of human beings perception of difference and similarity in semantic cognitions, accurate image annotation marked by semantic labels can work out the semantic gap puzzle [25] somewhat via whether professional labors or with the help of automatic image semantics annotation in which most of the time can be saved. And, the error rate of image annotation can be reduced distinctively, especially when the annotation amount is huge. Many valid methods for multi-instance [26, 27] and multi-label $[28,29,30]$ automatic image annotation have been studied and developed in recent years [31, 32, 33, 34, 35, 36]. Image annotation was usually being treated as problem of classifying [37], and was worked out through supervised learning mechanism or unsupervised learning one [38, 39, 40]. Methods based on machine learning [41], relevance feedback [42] and ontology [43] have been studied in depth. T. Kanimozhi and K. Latha integrated support vector machine based learning with an evolutionary stochastic algorithm, taking the firefly algorithm as a relevance feedback approach into a region based image retrieval system. It reduced the semantic gap through optimized iterative learning and also provided a better exploration of solution space [11]. M. R. B and J. L etc. proposed a framework to handle classes overlapping in features space for learning multi-label scene classification [26]. ML-kNN, a lazy learning method of multi-label learning was proposed by Zhang and Zhou [28], and, it was performed for automatic image annotation in natural image classification. And, the number of class label taken in their experiments [28] is only 1.24 for each image on average.

However, the semantics gap and the relation between image feature structure and semantic content itself remain still a big challenging problem for us.

For the sake of mining further the relation between image structural components difference and semantic similarity cognition, we proposed in this paper a method for multiple features fusion embodying rotation-invariant uniform LBP histogram distribution, weighted histogram integral and statistics of connected regions for automatic image annotation based on multi-label k-nearest neighbor algorithms in order to achieve better predicting performance. Both part of the technologies about content-based image retrieval and automatic image annotation were used and operated in a coordinated and complementary means in this paper. The main inherent image features are derived from mining and extraction of image content, in which the rotation-invariant uniform LBP histogram distribution, the weighted histogram integral and statistical information of connected regions are three main additional important components brought by us during the fusion of different compound image features. The multi-label learning method is used to realize automatic image annotation. The work in this paper was on the basis of multi-feature fusion method for automatic multilabel image annotation[44] and part of its processing were updated and extended mainly by newly introducing of another 18- dimension rotation-invariant uniform LBP histogram distribution features. We made comparisons among three different dimensional features fusions and implemented automatic image annotation experiments to compare them with multiple performance indexes. The total dimension numbers are individually set to 273, 17 and 35 in this paper. We compared among these three different methods including three different feature sets to investigate the impacts of different composite features in image in automatic image annotation. Different compound information of color, texture distribution, rotation-invariant uniform local binary pattern histogram distribution, and statistics of connected regions in image were combined together and were utilized in feature fusion and automatic image annotations were implemented based on multi-label learning algorithm.

\section{Method}

\subsection{Performance measurement index for multi-label learning}

In traditional supervised learning, each instance was just treated as belonging only to one class. But actually, an instance is often related closely to two or multi-class in real life. In the classification of images, each image has different regional objects representing multiple different semantics.

Function 'f 'was derived from learning process, $\mathrm{f}: \mathrm{x} \rightarrow 2^{Y}$. In mapping of $\mathrm{s}:\left(\mathrm{x}_{1}, \mathrm{y}_{1}\right),\left(\mathrm{x}_{2}, \mathrm{y}_{2}\right), \cdots,\left(\mathrm{x}_{m}, \mathrm{y}_{m}\right), \mathrm{x}_{i}$ is one of the elements in $\mathrm{X}$ set which was used to represent one image in data set. And $y_{i}$ is the label set of $i$-th image and at the same time $\mathrm{y}_{i}$ is one sub set of the full label set Y.

The method of multi-label k-nearest neighbor [28] is adopted in this paper in which principle of maximum a posteriori was used to determine the label set for the image instance tested.

Hamming loss, one-error, coverage, ranking loss and average precision are proposed to be used as algorithm performance measurement index [31] in (1)(2)(3)(4)). The variant of $\mathrm{v}$ denotes the label set in both equation (3) and equation (4).

Hamming loss:

$$
\operatorname{hloss}_{S}(h)=\frac{1}{u} \sum_{i=1}^{u} \frac{1}{Q}\left|h\left(x_{i} \Delta Y_{i}\right)\right|
$$

One-error:

$$
\operatorname{OneEr}_{s}(H)=\frac{1}{u} \sum_{i=1}^{u}\left[\left[H\left(x_{i}\right) \notin Y_{i}\right]\right]
$$

Coverage:

$$
\operatorname{Covg}_{s}(H)=\frac{1}{u} \sum_{i=1}^{u} \max _{v \in Y_{i}} \operatorname{rank}_{f}\left(x_{i}, v\right)-1
$$

Average precision:

$$
\operatorname{avpr}_{s}(H)=\frac{1}{u} \sum_{i=1}^{u} \frac{1}{\left|Y_{i}\right|} \sum_{v \in Y_{i}} \frac{\left|\left\{v^{\prime} \in \mathrm{Y}_{\mathrm{i}} \mid \operatorname{rank}_{\mathrm{f}}\left(\mathrm{x}_{\mathrm{i}}, v^{\prime}\right) \leq \operatorname{rank}_{\mathrm{f}}\left(\mathrm{x}_{\mathrm{i}}, v\right)\right\}\right|}{\operatorname{rank}_{f}(x, v)}
$$




\subsection{Color space transformation and weighted gray histogram integral}

In HSL [16] space, hue, saturation, and lightness, and at the same time correspondingly, hue, saturation, value in HSV space are essential variants to characterize the image. For the sake of focusing on and grasping the main distribution information, transformation of color image from RGB color space to gray level space can be implemented. And histogram vector was often used to illustrate the distribution of basic color. Gray histogram, the statistical numbers of each different gray level from 0 to 255 (8-bits) of the i-th picture was represented by the feature vector: $\left(\mathrm{N}_{i 0}, \mathrm{~N}_{i 1}, \mathrm{~N}_{i 2}, \cdots, \mathrm{N}_{i 255}\right)$. So, the picture set are represented in the form of gray histogram vectors set. The distance between two different pictures can be measured by similarity degree between their corresponding image gray histogram vectors, such as comparing them by calculating the inner product, angles cosine ratio. The matrices should be normalized ahead of time. Besides image gray histogram, many features also can be represented by means of histogram. Color histogram, Hue, Saturation, Lightness, or Value histogram in HSL or HSV space, transferred domain space histogram, such as in DFT (discrete fourier transform), DCT (discrete cosine transform), DWT (discrete wavelet transform) frequency domains. The frequency domain vectors are gotten accordingly. In order to keep more original and local information of true distribution, the neighborhood coherent pixel numbers should be taken into account which can enhance the statistical information amount and thereby the coherent pixel numbers vector was a meaningful supplement to the histogram distribution.
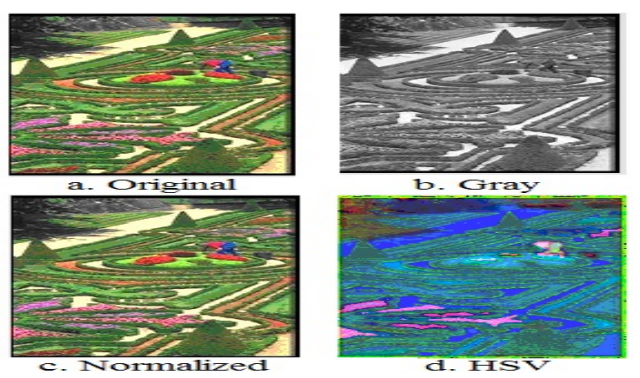

Figure 1: Color space transformation

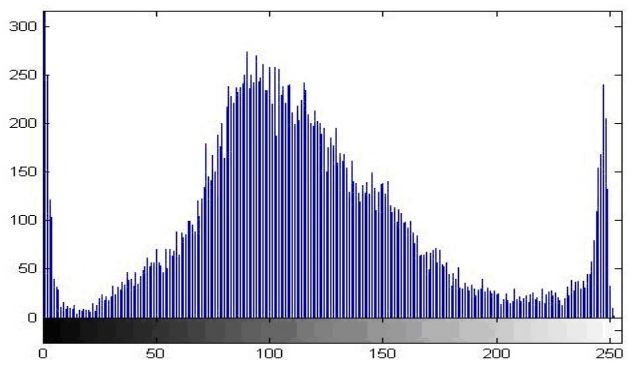

Figure 2: Histogram of gray level (Image No.:102057)

In order to reduce the dimensions of features vector, the discrete histogram distribution information of each gray level were replaced by weighted histogram integral area in which gray level is the weight of corresponding frequency (shown in (5)).

$$
S_{\text {Hist }}=\sum_{i=0}^{255}(i+1) f(i)
$$

Therefore, the vector of $\left(\mathrm{N}_{i 0}, \mathrm{~N}_{i 1}, \mathrm{~N}_{i 2}, \cdots, \mathrm{N}_{i 255}\right)$ can be replaced by weighted histogram integral area $\mathrm{S}_{\text {Hist }}$.

\subsection{Rotation-invariant uniform LBP histogram distribution}

The value of basic local binary pattern (LBP) of a pixel $(\mathrm{x}, \mathrm{y})$ is given by (6) and (7). In this paper, the $\mathrm{P}$ is $16, \mathrm{R}$ is 2 and rotation-invariant uniform LBP [45] histogram distribution information was utilized to be treated as another new 18 dimensional features data in one image.

$$
L B P_{P, R}=\sum_{p=0}^{P-1} S\left(g_{p}-g_{c}\right) 2^{p}
$$

where, $\mathrm{g}_{c}$ stands for the central pixel value and $\mathrm{g}_{p}$ denotes the pixel value of the $\mathrm{p}$-th neighbor pixel related to the central one.

And,

$$
S(x)=\left\{\begin{array}{lll}
1, & \text { if } & x \geq 0 \\
0, & \text { if } \quad x<0
\end{array}\right.
$$

As far as uniform LBP mode was concerned and used in this paper, the total transformation times of changing including whether from 0 to 1 or from 1 to 0 are less than and equal to 2 .

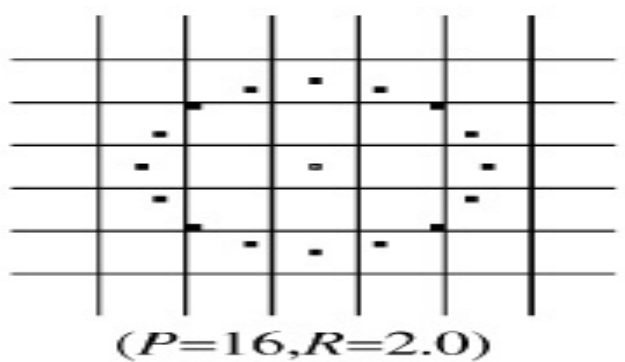

Figure 3: $\operatorname{LBP}_{P}{ }^{R}$ operator $\left(\mathrm{LBP}_{16}{ }^{2}\right)$

\subsection{Statistical information of connected regions}

There are more than two and three labels for most of the images in Corel5k image data set. One label is clearly correspondent to one specific semantics accordingly. Implicit relation between semantics and image regions of interest is assumed to be mined by utilizing of rotation-invariant uniform LBP histogram distribution, counting the region numbers and the total areas of connected regions and computation of gray level histogram integral in this paper.

As usual, one separate region of interest (ROI) in image represents one concrete semantic target in the image. Each individual target region can be detected by edge detection method. The Canny edge detection operator was utilized in the experiments. 
$\mathrm{N}_{R O I}$ and $\mathrm{S}_{\text {closure }}$ are the representative symbols of number of connected ROI and total areas of connected quarters in image respectively. And the vector $\left(\mathrm{N}_{R O I}, \mathrm{~S}_{\text {closure }}\right)$ was the comprehensive features of one independent interest region or target in one image.

Taking the coarse edge detection as input at low level implemented by differential operator, i.e. Canny edge detection operator, the computing of closure characteristics was conducted among potential target region contour edges on the basis of different and typical relations existed in closure topology [46] in order to judge the relations among various edges.
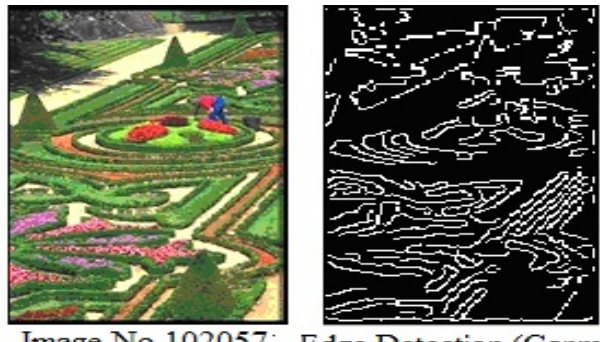

Edge Detection (Canny)

Figure 4: Edge detection (Canny)

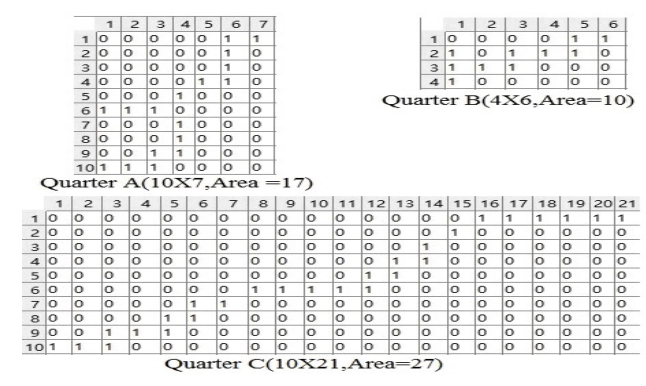

Figure 5: Statistical information of connected regions

Counting the number of closure contours or outlines closed and calculating the total closure area connected, both of them were taken into account being treated as the new features. As usual, one separate region of interest represents part of the concrete component of semantic target in one image after edge detection. At the same time, each individual whole target region can be described in one semantic word in the form of textual label.

The number of closure and connected regions in one image represents the discrete distribution that contains the configuration information in the perspective of composition.

Total area of closure and connected regions in one image in this paper is calculated by counting the whole pixel number of these closure and connected regions in one image after the operation of edge detection and binarization. Different quarters in Fig. 5 stand for different connected regions in one same image. The symbolic mark of 'Quarter A' means the first connected region while symbolic mark of 'Quarter B' and 'Quarter C' represent the second and the third one in the same image. And, the areas are their corresponding summaries of pixels connected to each other in individual region connected.

\subsection{Features fusion of color moments and texture information}

In one image, n-order color moments of all the sub-color variants can be used as important feature vectors.

$$
\begin{gathered}
\mu_{R}=\frac{1}{N} \sum_{i=1}^{N} P_{i R} \quad, \mu_{G}=\frac{1}{N} \sum_{i=1}^{N} P_{i G}, \mu_{B}=\frac{1}{N} \sum_{i=1}^{N} P_{i B} \\
\sigma_{R}=\sqrt{\frac{\sum_{i=1}^{N}\left(P_{i R}-\mu_{R}\right)^{2}}{N}}, \sigma_{G}=\sqrt{\frac{\sum_{i=1}^{N}\left(P_{i G}-\mu_{G}\right)^{2}}{N}}, \sigma_{\mathrm{B}}=\sqrt{\frac{\sum_{i=1}^{N}\left(P_{i B}-\mu_{B}\right)^{2}}{N}}
\end{gathered}
$$

$$
S_{R}=\sqrt[3]{\frac{\sum_{i=1}^{N}\left(P_{i R}-\mu_{R}\right)^{3}}{N}}, S_{G}=\sqrt[3]{\frac{\sum_{i=1}^{N}\left(P_{i G}-\mu_{G}\right)^{3}}{N}}, S_{B}=\sqrt[3]{\frac{\sum_{i=1}^{N}\left(P_{i B}-\mu_{B}\right)^{3}}{N}}
$$

Where, $\mathrm{N}$ is the total pixel numbers of the image in (8), $\mu_{R}, \mu_{G}, \mu_{B}$ are 1-order color moment which stand for the mean value of all pixel. And $\sigma_{R}, \sigma_{G}, \sigma_{B}, \mathrm{~S}_{R}, \mathrm{~S}_{G}, \mathrm{~S}_{B}$ are accordingly the 2-order and 3-order color moments.

If only 1, 2, 3-order moments are taken into account, one image features vector can be appended by one 9 dimensional vectors: $\left(\mu_{R}, \mu_{G}, \mu_{B}, \sigma_{R}, \sigma_{G}, \sigma_{B}, \mathrm{~S}_{R}, \mathrm{~S}_{G}, \mathrm{~S}_{B}\right)$

1 -order moment is the mean value of color. 2-order moment represents variance and 3-order moment describes the color bias.

Multiple different features were combined into one comprehensive vector in order to improve the image annotation accuracy for content-based image retrieving. Making full use of the fusion of gray histogram or HSL, HSV color histogram or pixel coherent numbers histogram and color moment, the new compound feature vector can be appended to describe part of information about original image in time domain.

Tamura texture features, different kinds of sub parameters including roughness, contrast, direction, resolution lines, regulation and coarseness are often used to illustrate the texture of image according to the visual psychology $[16,17]$.

Image was transformed from color to gray one, given that total level is 256 , then, the gray symbiosis matrix $M(a, b)$ is the matrix which has 256 rows and columns. The element of gray symbiosis matrix $\mathrm{M}(\mathrm{a}, \mathrm{b})(\mathrm{i}, \mathrm{j})$ denotes that two different pixel whose gray level is $i$ and $j$ individually, and at the same time, the position difference between their subscript are corresponding a and $b$. The values of the matrix elements stand for the times of couple pixels occurred after the whole retrieval for matrix was finished. Gray symbiosis matrix [16] describes the space distribution of gray level in image. The minimum the texture, the least the value of $a$ and $b$ should be.

$$
M_{i j}=\frac{M_{i j}}{\sum_{i} \sum_{j} M_{i j}}
$$

And, $\mathrm{J}$ is the energy.

$$
\mathbf{J}=\sum_{i} \sum_{j} M_{i j}^{2}
$$


$\mathrm{G}$ is inertia moment.

$$
G=\sum_{i} \sum_{j}(i-j)^{2} M_{i j}
$$

$\mathrm{S}$ is the entropy.

$$
S=-\sum_{i} \sum_{j} M_{i j} \log \left(M_{i j}\right)
$$

$\mathrm{C}$ is the coherence.

$$
C=\frac{\sum_{i} \sum_{j} i j M_{i j}-\mu_{a} \mu_{b}}{\sigma_{a}^{2} \sigma_{b}^{2}}
$$

Where,

$$
\begin{aligned}
& \mu_{a}=\sum_{i} i \sum_{j} M_{i j}, \mu_{b}=\sum_{j} j \sum_{i} M_{i j} \\
& \sigma_{a}{ }^{2}=\sum_{i}\left(i-\mu_{a}\right)^{2} \sum_{j} M_{i j}, \sigma_{b}{ }^{2}=\sum_{j}\left(j-\mu_{b}\right)^{2} \sum_{i} M_{i j}
\end{aligned}
$$

Because $\mathrm{J}, \mathrm{G}, \mathrm{S}, \mathrm{C}$ are all the functions of $\mathrm{M}_{i j}$ (gray symbiosis matrix) which describes the texture distribution of gray level in image. And, J, G, S, C are different aspects of texture distribution, so they are used jointly to represent the texture distribution information of a image. The texture feature vector $(\mathrm{J}$, $\mathrm{G}, \mathrm{S}, \mathrm{C}$ ) is used to illustrate the image texture distribution characteristics.

\subsection{Measurement of distance between two different vectors}

Both inner-product and distance can be used to measure the degree of difference between two different images compared to each other.

$$
D(X, Y)=\sum_{i=1}^{Z} \omega_{i} x_{i} y_{i}
$$

Where, $\mathrm{Z}$ is the total dimensional numbers and $\omega_{i}$ is $\mathrm{i}$-th dimensional weight. And $\mathrm{x}_{i}, \mathrm{y}_{i}$ are individually the $\mathrm{i}$-th sub variant value of image $\mathrm{X}$ and $\mathrm{Y}$.

$$
\begin{aligned}
& D(X, Y)=\left(\sum_{i=1}^{Z} \omega_{i}\left|x_{i}-y_{i}\right|^{p}\right)^{\frac{1}{p}} \\
& D(X, Y)=\frac{\sum_{i=1}^{Z} \min \left(x_{i}, y_{i}\right)}{\min \left(\sum_{i=1}^{Z} x_{i}, \sum_{i=1}^{Z} y_{i}\right)} \\
& D(X, Y)=(X-Y) C(X-Y)^{T}
\end{aligned}
$$

And, $\mathrm{C}$ presents the represents the covariance matrix.

When the value of $\mathrm{p}$ is 2 in (17), the distance is called Euclidean distance [25] which was adopted in this paper. As far as different features are concerned, their contributions to essence of images are different. Different weight combination represents different distribution of contributions among different features of image.

$$
d_{i}=\sum_{k=1}^{Z} \omega_{k} d_{i k}
$$

Where, $\mathrm{Z}$ is the total number of features types and the means of equal weight was adopted in this paper.

\section{Result and analysis}

\subsection{Data set}

Free version of part of Corel5K image data set was used for the experiments in this paper. The size format of image are 128, 192 pixels individually.

Total numbers of labels are 260 and each image has about 15 annotations or labels averagely. The label set attached to both train set and test set in our experiments is composed of 260 different labels, that is city, mountain, sky, sun, water, clouds, tree, lake, sea, beach, boats, people, branch, leaf, grass, palm, horizon, hills, waves, birds, $\cdots$.

Numbers of images in train set and test set are accordingly 4500 and 499.

\subsection{Features vector components of image}

Five parts are included in features vector and they are listed in detail as the following:

- The number, total areas of connected regions after edge detection.

- The rotation-invariant uniform LBP histogram distribution.

- Mean value, standard deviation and 3-order color moments.

- Texture information, contrast, correlation, energy, homogeneity, total gray common matrix energy (HSV color space).

- Gray histogram of image.

\subsection{Experimental results}

The original images in data set were transformed from color image to gray one and HSV color space separately. The texture information characterized by contrast, correlation, energy, homogeneity and total gray common matrix energy in HSV color space was listed in Table 1. Color moment information characterized by the following n-order variants including mean value, standard deviation and 3-order color moment were given in Table 2 and Table 3 separately. Both 3 columns of result data came accordingly from unnormalized image (in Table 2.) and normalized one (in Table 3.).

In the process of multi-label image semantics prediction, the changing of average precision of prediction on test data set with the adjustment and modification of parameter $\mathrm{K}$ which was used to represent the numbers of nearest neighbor were recorded in line chart of Fig. 11.

Table 2: Unnormalized color moment features (Image No.: 102057)

\begin{tabular}{cccc}
\hline Sub color & Mean & Standard deviation & 3-order color moment \\
\hline R & 114.7486 & 64.2621 & 55.7801 \\
G & 123.4308 & 56.3803 & 38.6501 \\
B & 77.8222 & 46.2671 & 44.8914 \\
\hline
\end{tabular}


Table 1: Image texture features

\begin{tabular}{cccccc}
\hline Image No. & Contrast & Correlation & Energy & Homogeneity & Total gray common matrix energy \\
\hline 102057 & 153.1710 & 2.3906 & 0.0194 & 1.6302 & 11.5225 \\
\hline
\end{tabular}

Table 5: Comparison of performance index in automatic image annotation by MLKNN $(\mathrm{K}=18$, smooth=1)

\begin{tabular}{cccccc}
\hline Feature Dimensions & Coverage & Hamming Loss & Ranking Loss & One-Error & Average precision \\
\hline 273 & 94.6493 & 0.9993 & 0.1592 & 0.6713 & 0.2898 \\
17 & 86.1483 & 0.9989 & 0.1396 & 0.5992 & 0.3518 \\
35 & 79.8697 & 0.9985 & 0.1273 & 0.5291 & 0.3954 \\
\hline
\end{tabular}

Table 3: normalized color moment features (Image No.: 102057)

\begin{tabular}{cccc}
\hline Sub color & Mean & Standard deviation & 3-order color moment \\
\hline R & 0.4500 & 0.2520 & 0.2187 \\
G & 0.4840 & 0.2211 & 0.1516 \\
B & 0.3176 & 0.1888 & 0.1832 \\
\hline
\end{tabular}

Table 4: Number of all connected quarters and total areas of connected regions

\begin{tabular}{cccc}
\hline Image No. & Image Size & Quarters Number & Total Areas \\
\hline 102057 & $192 \times 128$ & 79 & 3961 \\
\hline
\end{tabular}

Final experimental results of prediction were given in Table 5 with the help of measure index of hamming loss, one-error, coverage, ranking loss and average precision. The total dimensions of feature vectors are correspondingly 273, 17 and 35 in three times of experiments under different conditions. In all experiments, all the images pixel data in train set and test set were normalized to 01 . In the third experiment, the components of image features vector with 35-dimension features were finally listed in sequence as the following: number of connected regions, total areas of region connected, weighted histogram area, HSV spaces contrast, correlation, energy, homogeneity, total gray common matrix energy, 9-dimension features are RGB color spaces mean value, standard deviation, 3-order color moment and new 18 dimensional features data of rotation-invariant LBP histogram distribution information appended.

It showed that the prediction performance of automatic image annotation under the condition that image was normalized outperformed that of unnormalized one in Table 5. After removing the 256 separate and concrete gray level distribution information and replacing them with one single weighted histogram integral area, hence, the total dimensional degree of feature vectors were cut down from 273 to 17 , the performance of automatic multi-label image annotation was improved notably from 0.2898 to 0.3518 as far as the average precision measure index was concerned. At the same time, one-error was decreased from 0.6713 to 0.5992 , coverage was decreased from 94.6493 to 86.1483 and ranking loss was decreased from 0.1592 to 0.1396 . After appending another 18 -dimension rotationinvariant LBP histogram distribution information, the average precision was improved from 0.3518 to 0.3954 while coverage was decreased from 86.1483 to 79.8697 , hamming loss was var-

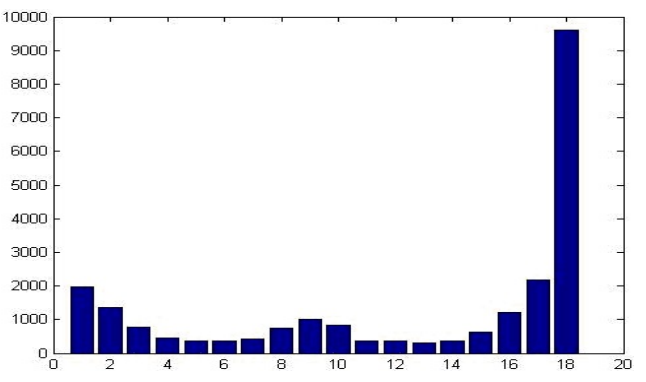

Figure 6: Bar of histogram distribution via rotation-invariant uniform $\operatorname{LBP}(p=16, R=2)$

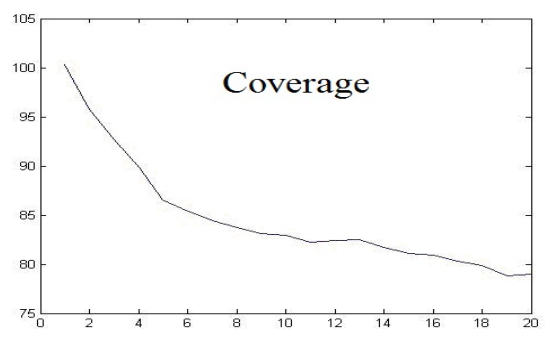

Figure 7: Coverage

ied from 0.9989 to 0.9985 , one-error was reduced from 0.5992 to 0.5291 and the ranking loss was decreased from 0.1396 to 0.1273 .

After the histogram integral area was taken the place of individual and discrete gray level histogram distribution, the concrete low weight features was gotten rid of, then the computing time was saved and the final predicting performance was improved at the same time. In order to transform and compress too much redundant and different gray level distributions information into one net and unique 1-dimension feature, we make the integral histogram area multiplied by different weight coefficients. The weighted histograms integral area is more unique with related to the original image distribution than the sum of histogram area without weight. Additionally, choosing the 18 dimensional rotation-invariant uniform LBP histogram distribution as one of the compound features data is a good supplemental means to feature fusion in the process of automatic image annotation via multi-label learning algorithm. The appending of rotation-invariant uniform LBP histogram distribution information in this paper enhanced the mining amount of image tex- 


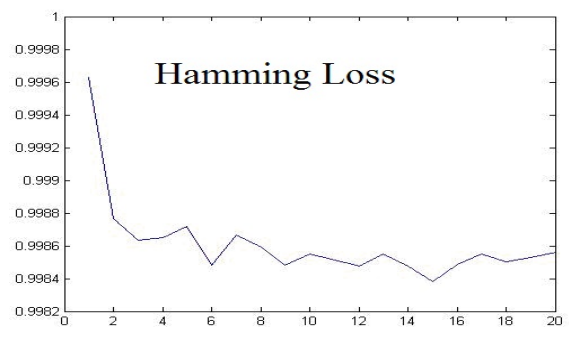

Figure 8: HammingLoss

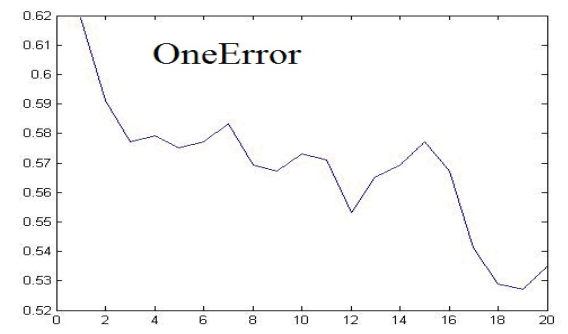

Figure 9: One-Error

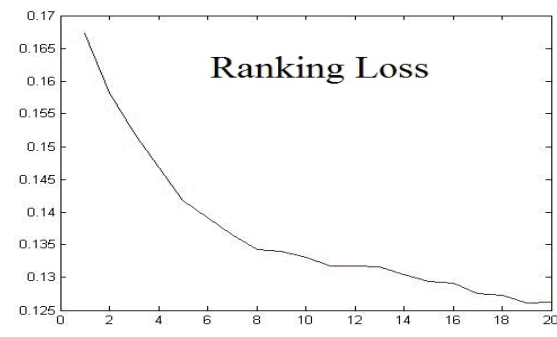

Figure 10: RankingLoss

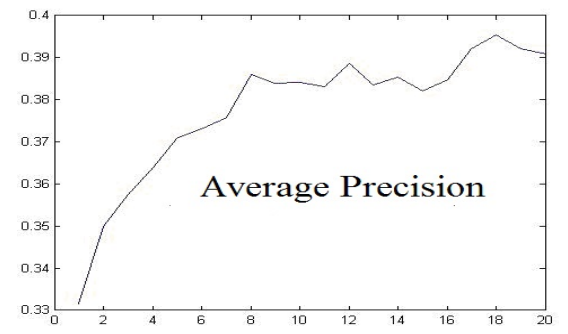

Figure 11: Average precision with the changing of parameter $\mathrm{K}$ in $\mathrm{KNN}$ ture information in which the predicting ability was improved.

But, in the perspective of comprehensive multi-label learning algorithms predicting performance, the experimental results are not very good. And to some extend, the predicting efficiency is a little low compared to some other ideal and successful multi-label learning experiments in the filed of automatic image annotation reported in some literature documents, i.e. [28]. The reason may exist in three aspects. Firstly, the computation of total areas of connected regions in image depends closely on the numbers counting of fragmental closure target. Too much separate connected regions possibly had separated the limited semantic bodies contained in image into many fragments. Secondly, many of the semantic label numbers in one image for the experimental data set used in this paper are over 4 which made the predicting more difficult. Lastly, the exploration and mining of relation between image feature data including LBP texture, statistical information of connected regions and that of images true semantic cognition are complicated and difficult.

\section{Conclusion}

Feature extraction, feature fusion, distance measurement, learning algorithms are key elements of automatic image annotation. To mine the quantitative impact of different image feature vectors on prediction of semantic labels in automatic image annotation, LBP texture information, statistics of connected regions and weighted histogram integral areas were fully utilized. Different experiments based on Corel5K image data base and multi-label learning k-nearest neighbor algorithm were conducted To improve the predicting performance and quality, a method for automatic image annotation with multi-feature fusion including rotation-invariant uniform LBP histogram distribution, weighted histogram integral areas and statistics of connected regions based on multi-label learning k-nearest neighbor algorithm was proposed in this paper. Relying only on the traditional n-order color moments and normal texture distribution information including contrast, correlation, energy, homogeneity, common gray matrix and discrete gray level histogram, the predicting performance can be improved limitedly. Reducing the dimension of feature vectors by cutting down less weight features, discrete and separate gray level information, time consuming can be decreased. Bringing new typical features into the process of feature fusion, including the rotation-invariant uniform LBP histogram distributions, the weighted histogram integral areas and statistical information of connected regions in image can improved the predicting performance. It showed that the proposed method for automatic image annotation has achieved $36.4 \%$ performance improvement from 0.2898 to 0.3954 on average precision.

\section{Acknowledgement}

This work was funded by the National Science Foundation of China (No.61472282, No.61300058 and No.61271098).

\section{References}

[1] J. C. Caicedo, J. BenAbdallah, F. A. Gonzlez, O. Nasraoui, Multimodal representation, indexing, automated annotation and retrieval of image collections via non-negative matrix factorization, Neurocomputing 76 (2012) 50 - 60. Seventh International Symposium on Neural Networks (ISNN 2010)Advances in Web Intelligence.

[2] D. D. Burdescu, C. G. Mihai, L. Stanescu, M. Brezovan, Automatic image annotation and semantic based image retrieval for medical domain, Neurocomputing 109 (2013) 33 - 48. New trends on Soft Computing Models in Industrial and Environmental ApplicationsA selection of extended and updated papers from the $\{\mathrm{SOCO}\} 2011$ International Conference. 
[3] L. Shang, D.-S. Huang, J.-X. Du, C.-H. Zheng, Palmprint recognition using fastica algorithm and radial basis probabilistic neural network, Neurocomputing 69 (2006) 1782-1786.

[4] L. Ogiela, Cognitive informatics in image semantics description, identification and automatic pattern understanding, Neurocomputing 122 (2013) $58-69$. Advances in cognitive and ubiquitous computingSelected papers from the Sixth International Conference on Innovative Mobile and Internet Services in Ubiquitous Computing (IMIS-2012).

[5] F. van der Sommen, S. Zinger, E. Schoon, P. de With, Supportive automatic annotation of early esophageal cancer using local gabor and color features, Neurocomputing 144 (2014) 92 - 106.

[6] A. F. D. Souza, F. Pedroni, E. Oliveira, P. M. Ciarelli, W. F. Henrique, L. Veronese, C. Badue, Automated multi-label text categorization with vg-ram weightless neural networks, Neurocomputing 72 (2009) 2209 2217. Lattice Computing and Natural Computing (JCIS 2007) / Neural Networks in Intelligent Systems Designn (ISDA 2007).

[7] E. de Ves, X. Benavent, I. Coma, G. Ayala, A novel dynamic multimodel relevance feedback procedure for content-based image retrieval, Neurocomputing (2016) -

[8] P. Shanmugavadivu, P. Sumathy, A. Vadivel, Fosir: Fuzzy-object-shape for image retrieval applications, Neurocomputing 171 (2016) $719-735$.

[9] K. Iqbal, M. Odetayo, A. James, R. Iqbal, N. Kumar, S. Barma, An efficient image retrieval scheme for colour enhancement of embedded and distributed surveillance images, Neurocomputing 174, Part A (2016) 413 -430 .

[10] J.-X. Mi, D.-S. Huang, B. Wang, X. Zhu, The nearest-farthest subspace classification for face recognition, Neurocomputing 113 (2013) 241 250.

[11] T. Kanimozhi, K. Latha, An integrated approach to region based image retrieval using firefly algorithm and support vector machine, Neurocomputing 151, Part 3 (2015) 1099 - 1111

[12] S. K. Vipparthi, S. Murala, S. Nagar, A. B. Gonde, Local gabor maximum edge position octal patterns for image retrieval, Neurocomputing 167 (2015) $336-345$.

13] I. G. P. S. Wijaya, K. Uchimura, G. Koutaki, Multi-pose face recognition using fusion of scale invariant features, in: Proceedings of the 2011 2nd International Congress on Computer Applications and Computational Science, Springer, 2012, pp. 207-213.

[14] J. Amadsun, R. King, Textural features corresponding to texture properties, IEEE Trans. Syst., Man, Cybern 19 (1989) 1264-1274.

[15] T. Chang, C. J. Kuo, Texture analysis and classification with treestructured wavelet transform, Image Processing, IEEE Transactions on 2 (1993) 429-441.

[16] Y.-J. Zhang, Image processing and analyzing technology, 2nd ed., Beijing: Higher education press, 2008.

[17] Y.-J. Zhang, Visual information retrieving based on content, Beijing: Science press., 2003

[18] R. Krishnamoorthi, S. S. Devi, A simple computational model for image retrieval with weighted multifeatures based on orthogonal polynomials and genetic algorithm, Neurocomputing 116 (2013) 165 - 181. Advanced Theory and Methodology in Intelligent ComputingSelected Papers from the Seventh International Conference on Intelligent Computing (ICIC 2011).

[19] K.-S. Lee, A. N. Rosli, I. A. Supandi, G.-S. Jo, Dynamic samplingbased interpolation algorithm for representation of clickable moving object in collaborative video annotation, Neurocomputing 146 (2014) 291 - 300. Bridging Machine learning and Evolutionary Computation (BMLEC)Computational Collective Intelligence.

[20] B. Li, C.-H. Zheng, D.-S. Huang, Locally linear discriminant embedding: An efficient method for face recognition, Pattern Recognition 41 (2008) 3813-3821

[21] Z.-Q. Zhao, D.-S. Huang, B.-Y. Sun, Human face recognition based on multi-features using neural networks committee, Pattern Recognition Letters 25 (2004) 1351-1358

[22] X.-F. Wang, D.-S. Huang, A novel density-based clustering framework by using level set method, Knowledge and Data Engineering, IEEE Transactions on 21 (2009) 1515-1531.

[23] X.-F. Wang, D.-S. Huang, H. Xu, An efficient local chan-vese model for image segmentation, Pattern Recognition 43 (2010) 603-618.

[24] J. J.-Y. Wang, H. Bensmail, X. Gao, Joint learning and weighting of visual vocabulary for bag-of-feature based tissue classification, Pattern
Recognition 46 (2013) 3249-3255.

[25] T. Hofmann, Probabilistic latent semantic indexing, in: Proceedings of the 22nd annual international ACM SIGIR conference on Research and development in information retrieval, ACM, 1999, pp. 50-57.

[26] M. R. Boutell, J. Luo, X. Shen, C. M. Brown, Learning multi-label scene classification, Pattern recognition 37 (2004) 1757-1771.

[27] M.-L. Zhang, Z.-H. Zhou, M3miml: A maximum margin method for multi-instance multi-label learning, in: Data Mining, 2008. ICDM'08. Eighth IEEE International Conference on, IEEE, 2008, pp. 688-697.

[28] M.-L. Zhang, Z.-H. Zhou, Ml-knn: A lazy learning approach to multilabel learning, Pattern recognition 40 (2007) 2038-2048.

[29] X.-S. Xu, Y. Jiang, L. Peng, X. Xue, Z.-H. Zhou, Ensemble approach based on conditional random field for multi-label image and video annotation, in: Proceedings of the 19th ACM international conference on Multimedia, ACM, 2011, pp. 1377-1380.

[30] X. Kong, M. K. Ng, Z.-H. Zhou, Transductive multilabel learning via label set propagation, Knowledge and Data Engineering, IEEE Transactions on 25 (2013) 704-719.

[31] R. E. Schapire, Y. Singer, Boostexter: A boosting-based system for text categorization, Machine learning 39 (2000) 135-168.

[32] J. J. Wang, H. Bensmail, X. Gao, Multiple graph regularized protein domain ranking, BMC bioinformatics 13 (2012) 307.

[33] X. Geng, C. Yin, Z.-H. Zhou, Facial age estimation by learning from label distributions, Pattern Analysis and Machine Intelligence, IEEE Transactions on 35 (2013) 2401-2412.

[34] R. Jin, S. Wang, Z.-H. Zhou, Learning a distance metric from multiinstance multi-label data, in: Computer Vision and Pattern Recognition, 2009. CVPR 2009. IEEE Conference on, IEEE, 2009, pp. 896-902.

[35] J.-Y. Wang, I. Almasri, X. Gao, Adaptive graph regularized nonnegative matrix factorization via feature selection, in: Pattern Recognition (ICPR), 2012 21st International Conference on, IEEE, 2012, pp. 963-966.

[36] M.-L. Zhang, Z.-H. Zhou, Multi-label learning by instance differentiation, in: AAAI, volume 7, 2007, pp. 669-674.

[37] J. Li, J. Z. Wang, Automatic linguistic indexing of pictures by a statistical modeling approach, Pattern Analysis and Machine Intelligence, IEEE Transactions on 25 (2003) 1075-1088

[38] G. Carneiro, A. B. Chan, P. J. Moreno, N. Vasconcelos, Supervised learning of semantic classes for image annotation and retrieval, Pattern Analysis and Machine Intelligence, IEEE Transactions on 29 (2007) 394-410.

[39] C. Yang, M. Dong, J. Hua, Region-based image annotation using asymmetrical support vector machine-based multiple-instance learning, in: Computer Vision and Pattern Recognition, 2006 IEEE Computer Society Conference on, volume 2, IEEE, 2006, pp. 2057-2063.

[40] P. Quelhas, F. Monay, J.-M. Odobez, D. Gatica-Perez, T. Tuytelaars, L. Van Gool, Modeling scenes with local descriptors and latent aspects, in: Computer Vision, 2005. ICCV 2005. Tenth IEEE International Conference on, volume 1, IEEE, 2005, pp. 883-890.

[41] J. Shi, J. Malik, Normalized cuts and image segmentation, Pattern Analysis and Machine Intelligence, IEEE Transactions on 22 (2000) 888-905.

[42] D.-H. Kim, S.-H. Yu, A new region filtering and region weighting approach to relevance feedback in content-based image retrieval, Journal of Systems and Software 81 (2008) 1525-1538.

[43] J. Fan, Y. Gao, H. Luo, Integrating concept ontology and multitask learning to achieve more effective classifier training for multilevel image annotation, Image Processing, IEEE Transactions on 17 (2008) 407-426.

[44] S. Xia, P. Chen, J. Zhang, X.-P. Li, B. Wang, A multi-feature fusion method for automatic multi-label image annotation with weighted histogram integral and closure regions counting, in: Advanced Intelligent Computing Theories and Applications, Springer, 2015, pp. 323-330.

[45] T. Ojala, M. Pietikäinen, T. Mäenpää, Multiresolution gray-scale and rotation invariant texture classification with local binary patterns, Pattern Analysis and Machine Intelligence, IEEE Transactions on 24 (2002) 971987.

[46] J. H. Elder, S. W. Zucker, Computing contour closure, in: Computer VisionECCV'96, Springer, 1996, pp. 399-412. 


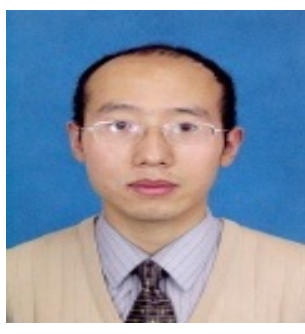

Sen Xia received the B.S degree from Xiamen University, Fujian, China in 1994 and received the M.S degree from Yunnan University, Kunming, China in 2005. He is now a Ph.D candidate majoring in computer science and technology in Tongji University, Shanghai, China. His research interests mainly focus on cognition and intelligent information processing.

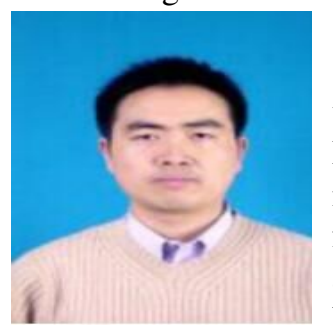

Pen Chen is a Professor in the Institute of Health Sciences, Anhui University, Hefei, China. He received his Bachelor degree from Electronic Engineering Institute, PLA, Master degree from Kunming University of Science and Technology, and Ph.D degree from University of Science and Technology of China. Prior to joining Anhui University, he served in City University of Hong Kong (2006, as senior research associate), Howard University (USA, 2008-2009, as Postdoc Fellow), Nanyang Technological University (Singapore, 20092010, as Research fellow), and King Abdullah University of Science and Technology (KAUST), Saudi Arabia (2012-2014, as Postdoc Fellow). From 2011 to 2013, he was an Associate Professor in Hefei Institute of Intelligent Machines, Chinese Academy of Sciences, China. He speciliases in machine learning and data mining with applications to bioinformatics, drug discovery, computer vision, etc. He has published about 50 high quality referred papers in international conferences and journals.

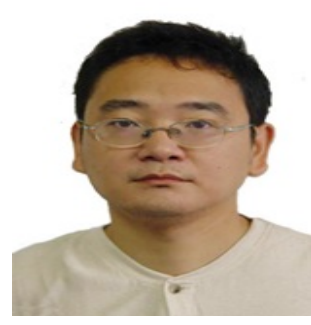

Jun Zhang was born in Anhui Province, Chin, in 1971. He received M.S. degree in Pattern Recognition \& Intelligent System in 2004, from Institute of Intelligent Machines, Chinese Academy of Sciences. He received the Ph.D degree from University of Science and Technology of China, Hefei, China in 2007. Currently, Dr. Zhang is associate professor in the School of Electrical Engineering and Automation, Anhui University, China. His research interests focus on deep learning, ensemble learning and cheminformatics.

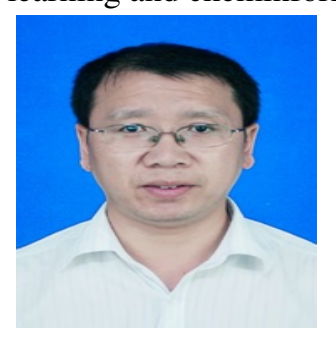

Xiaoping $\mathbf{L i}$ received the B.S degree from Jiangxi Institute of Education, Nanchang, Jiangxi, China in 2002 and received the M.S degree from Huazhong University of sciense and Technology, Wuhan, Hubei, China in 2006. His research interests mainly focus on information security and formal language.

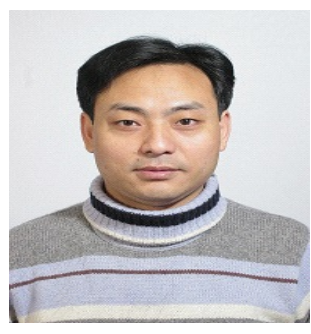

Bing Wang received the B.S. and M.S. degrees from Hefei University of Technology, Hefei, China in 1998 and 2004 respectively. He received the $\mathrm{Ph} . \mathrm{D}$ degree from University of Science and Technology of China, Hefei in 2006. Currently, Dr. Wang is serving as research professor in the School of Electronics and Information Engineering, Tongji University, Shanghai, China. His research interests mainly focus on machine learning, computational biology, and cheminformatics. 